\title{
Zeros of the Riemann Zeta-Function on the Critical Line
}

\author{
D.R. Heath-Brown \\ Magdalen College, Oxford
}

It was shown by Selberg [3] that the Riemann Zeta-function has at least $c T \log T$ zeros on the critical line up to height $T$, for some positive absolute constant $c$. Indeed Selberg's method counts only zeros of odd order, and counts each such zero once only, regardless of its multiplicity. With this in mind we shall write $\hat{\gamma}_{i}$ for the distinct ordinates of zeros of $\zeta(s)$ on the critical line of odd multiplicity. We shall number the points $\hat{\gamma}_{i}$ so that $0<\hat{\gamma}_{1}<\hat{\gamma}_{2}<\ldots$ The purpose of the present note is to extract a little more from Selberg's argument, by obtaining further information on the distribution of the $\hat{\gamma}_{i}$. This is given in the following result.

Theorem For any constant $\mu \in(0,2)$ we have

$$
\sum_{\hat{\gamma}_{i} \leq T}\left(\hat{\gamma}_{i+1}-\hat{\gamma}_{i}\right)^{\mu} \ll_{\mu} T(\log T)^{1-\mu} .
$$

In particular, if $f(T)$ is any function which tends to infinity with $T$, then "almost all" intervals $\left[T, T+f(T)(\log T)^{-1}\right]$ contain a point $\hat{\gamma}_{i}$.

Clearly this result includes Selberg's. Moreover it is apparent that the second statement of the theorem follows from the first. We also remark that, if one merely sums over ordinates $\gamma_{i}$ of the zeros in the usual sense, not restricting to those zeros which are on the critical line, then one has

$$
\sum_{\gamma_{i} \leq T}\left(\gamma_{i+1}-\gamma_{i}\right)^{\mu} \ll_{\mu} T(\log T)^{1-\mu}
$$

for any $\mu>0$, as was shown by Fujii [1].

In giving the proof of our result we shall refer to the version of Selberg's argument presented by Titchmarsh [4: $\S \S 10.9-10.22]$. The proof uses a "mollifier"

$$
\phi(s)=\sum_{\nu \leq X} \beta_{\nu} \nu^{-s},
$$


in which the numbers $\beta_{\nu}$ are defined in terms of the coefficients $\alpha_{\nu}$ in the expansion

$$
\zeta(s)^{-1 / 2}=\sum_{\nu=1}^{\infty} \alpha_{\nu} \nu^{-s} \quad \sigma>1
$$

Titchmarsh takes

$$
\beta_{\nu}=\alpha_{\nu}\left(1-\frac{\log \nu}{\log X}\right)
$$

but for our purpose the choice

$$
\beta_{\nu}=\left\{\begin{array}{cc}
\alpha_{\nu}, & \nu \leq X^{1 / 2} \\
2 \alpha_{\nu} \frac{\log X / \nu}{\log X}, & X^{1 / 2} \leq \nu \leq X
\end{array}\right.
$$

is required. One then defines

$$
F(t)=\frac{\Xi(t)}{t^{2}+\frac{1}{4}}\left|\phi\left(\frac{1}{2}+i t\right)\right|^{2} e^{\left(\frac{1}{4} \pi-\frac{1}{2} \delta\right) t},
$$

where $\delta$ is small and positive. In fact we shall take $\delta=T^{-1}$, where $[T, 2 T]$ is the interval in which we are looking for zeros. With the above definition of $F(t)$ it follows (Titchmarsh [4: Lemma 10.17]) that

$$
\int_{-\infty}^{\infty}\left|\int_{t}^{t+h} F(u) d u\right|^{2} d t \ll \frac{h}{\delta^{1 / 2} \log X}
$$

This is subject to the conditions $X=\delta^{-c}$ and $h=(a \log X)^{-1}$, where $a, c$ are positive and satisfy $(a+2) c \leq \frac{1}{4}$. Titchmarsh takes $a$ to be constant, but this is unnecessary. We shall set $c=\frac{1}{16}$ so that any value $a \in(0,2)$ is permissable. We see from (1) that

$$
\int_{T}^{2 T}\left|\int_{t-h}^{t+h} F(u) d u\right|^{2} d t \ll \frac{h T^{1 / 2}}{\log T},
$$

on changing $h$ into $2 h$ and substituting $t-h$ for $t$. The bound (2) is subject to the conditions $X=T^{1 / 16}$ and $h=\left(a^{\prime} \log T\right)^{-1}$, where $a^{\prime} \in\left(0, \frac{1}{4}\right)$.

The proof of (1), and hence of (2), depends on the definition of $\beta_{\nu}$, so we must check that our modification does not materially alter the estimates. It is only Lemma 10.12 of Titchmarsh [4] which needs any change. It is shown that

$$
\sum_{\kappa \leq X / d} \alpha_{\kappa} \kappa^{\theta-1} \log \frac{X}{d \kappa} \ll\left(\frac{X}{d}\right)^{\theta}\left(\log \frac{X}{d}\right)^{1 / 2} \prod_{p \mid \rho}\left(1+p^{-1}\right)^{1 / 2}
$$


uniformly for $0<\theta \leq \frac{1}{2}$, where $\kappa$ is restricted to integers coprime to $\rho$. We shall require a corresponding estimate in which the function

$$
f(X, d, \kappa)=\left\{\begin{array}{cc}
\log X / d \kappa, & d \kappa \leq X \\
0, & d \kappa \geq X
\end{array}\right.
$$

on the left is replaced by

$$
g(X, d, \kappa)=\left\{\begin{array}{cc}
\log X, & d \kappa \leq X^{1 / 2}, \\
2 \log X / d \kappa, & X^{1 / 2} \leq d \kappa \leq X, \\
0, & d \kappa \geq X .
\end{array}\right.
$$

However, since

$$
g(X, d, \kappa)=2 f(X, d, \kappa)-2 f\left(X^{1 / 2}, d, \kappa\right),
$$

one sees that (3) remains true with $g$ in place of $f$.

We shall also require the estimate

$$
\int_{-\infty}^{\infty}|F(t)|^{2} d t \ll \frac{\log 1 / \delta}{\delta^{1 / 2} \log X} \ll T^{1 / 2}
$$

given by Lemma 10.18 of Titchmarsh [4]. The proof of this requires no modification.

We now establish a lower bound for

$$
\int_{t-h}^{t+h}|F(u)| d u=J(t),
$$

say, on the interval $T \leq t \leq 2 T$. Titchmarsh does this only on average, while we shall, in effect, obtain a lower bound for "almost all" $t$. We begin by choosing a large constant integer $K$, and writing

$$
w(z)=\left(\frac{\sin z}{z}\right)^{2 K}
$$

so that

$$
\int_{-\infty}^{\infty} e^{i \lambda t} w(t) d t=0 \text { for }|\lambda| \geq 2 K .
$$

We now consider the integral

$$
\int_{1 / 2-i \infty}^{1 / 2+i \infty} \zeta(s+i t) \phi(s+i t)^{2} w\left(\frac{s-\frac{1}{2}}{i \Delta}\right) d s=I,
$$

say, where

$$
\frac{1}{\log T} \leq \Delta \leq T^{3 / 4}
$$


The integral will converge if $K$ is chosen large enough. We now move the line of integration to $\sigma=2$, producing a residue

$$
\phi(1)^{2} w\left(\frac{\frac{1}{2}-i t}{i \Delta}\right) \ll(\log T)^{2}\left(\frac{e^{1 / 2 \Delta}}{|t| / \Delta}\right)^{2 K} \ll(\log T)^{-2} .
$$

On the line $\sigma=2$ we may integrate termwise. We have

$$
\int_{2-i \infty}^{2+i \infty} n^{-s} w\left(\frac{s-\frac{1}{2}}{i \Delta}\right) d s=i \Delta \int_{-\infty}^{\infty} n^{-1 / 2-i t \Delta} w(t) d t
$$

on moving the line of integration back to $\sigma=1 / 2$, so that terms for which $\Delta \log n \geq 2 K$ make no contribution. Since

$$
\zeta(s) \phi(s)^{2}=\sum_{n=1}^{\infty} a_{n} n^{-s}
$$

with $a_{1}=1$ and $a_{n}=0$ for $2 \leq n \leq X^{1 / 2}$, we now see that

$$
I=i \Delta C_{K}+O\left(\frac{1}{\log ^{2} T}\right)
$$

where

$$
C_{K}=\int_{-\infty}^{\infty} w(t) d t>0
$$

providing that

$$
\Delta \geq \frac{64 K}{\log T}
$$

At this point we observe that if $h \leq T^{3 / 4}$ then

$$
\begin{aligned}
T^{1 / 4} J(t) & \gg \int_{t-h}^{t+h}\left|\zeta\left(\frac{1}{2}+i u\right) \phi\left(\frac{1}{2}+i u\right)^{2}\right| d u \\
& \geq \int_{t-h}^{t+h}\left|\zeta\left(\frac{1}{2}+i u\right) \phi\left(\frac{1}{2}+i u\right)^{2}\right| w\left(\frac{u}{\Delta}\right) d u \\
& \geq I+O\left\{\int_{|u| \geq h}\left|\zeta\left(\frac{1}{2}+i(u+t)\right) \phi\left(\frac{1}{2}+i(u+t)\right)^{2}\right| \frac{d u}{(|u| / \Delta)^{2 K}}\right\},
\end{aligned}
$$

whence

$$
T^{1 / 4} J(t)+\int_{|u| \geq h}\left|\zeta\left(\frac{1}{2}+i(u+t)\right) \phi\left(\frac{1}{2}+i(u+t)\right)^{2}\right| \frac{d u}{(|u| / \Delta)^{2 K}} \gg \Delta .
$$

Since

$$
\zeta\left(\frac{1}{2}+i(u+t)\right) \phi\left(\frac{1}{2}+i(u+t)\right)^{2} \ll(T+|u|)^{1 / 4} X
$$


for $T \leq t \leq 2 T$, it follows that the range $|u| \geq T / 2$ will contribute only $O(\Delta / T)$, say. Here we use the facts that $\Delta \leq T^{3 / 4}$, by (6), and that $K$ is sufficiently large. Moreover

$$
\begin{aligned}
\int_{h \leq|u| \leq T / 2} \mid \zeta\left(\frac{1}{2}\right. & +i(u+t)) \phi\left(\frac{1}{2}+i(u+t)\right)^{2} \mid(|u| / \Delta)^{-2 K} d u \\
& \ll T^{1 / 4} \int_{h \leq|u| \leq T / 2}|F(t+u)|(|u| / \Delta)^{-2 K} d u \\
& =T^{1 / 4} K(t),
\end{aligned}
$$

say. It follows that

$$
J(t)+K(t) \gg T^{-1 / 4} \Delta .
$$

We now observe that

$$
\int_{T}^{2 T} K(t) d t=\int_{h \leq|u| \leq T / 2}(|u| / \Delta)^{-2 K}\left\{\int_{T+u}^{2 T+u}|F(v)| d v\right\} d u
$$

and Cauchy's inequality, in conjunction with (4) yields

$$
\int_{T+u}^{2 T+u}|F(v)| d v \ll T^{1 / 2}\left\{\int_{T / 2}^{5 T / 2}|F(v)|^{2} d v\right\}^{1 / 2} \ll T^{3 / 4} .
$$

We therefore see that

$$
\int_{T}^{2 T} K(t) d t \ll \frac{h T^{3 / 4}}{(h / \Delta)^{2 K}},
$$

since

$$
\int_{h \leq|u| \leq T / 2}(|u| / \Delta)^{-2 K} d u \ll \frac{h}{(h / \Delta)^{2 K}} .
$$

We shall write (8) as

$$
J(t)+K(t) \geq C T^{-1 / 4} \Delta,
$$

and define

$$
R_{h}=\left\{t \in[T, 2 T]: J(t) \leq \frac{C}{2} T^{-1 / 4} \Delta\right\} .
$$

Then $K(t) \gg T^{-1 / 4} \Delta$ on $R_{h}$, whence

$$
T^{-1 / 4} \Delta \operatorname{mes}\left(R_{h}\right) \ll \frac{h T^{3 / 4}}{(h / \Delta)^{2 K}},
$$

by (9). It follows that

$$
\operatorname{mes}\left(R_{h}\right) \ll \frac{T}{(h / \Delta)^{2 K-1}} .
$$


We can now complete the proof of the theorem. We consider the set $S_{h}$ of $t \in[T, 2 T]$ for which the interval $t-h \leq u \leq t+h$ contains no sign change of the function $F(u)$, or equivalently no zero of odd order of $\zeta\left(\frac{1}{2}+i u\right)$. Thus

$$
J(t)=\left|\int_{t-h}^{t+h} F(u) d u\right|
$$

for $t \in S_{h}$. If $t \in S_{h} \backslash R_{h}$ then $J(t) \geq \frac{C}{2} T^{-1 / 4} \Delta$, whence (2) yields

$$
\left(\frac{C}{2} T^{-1 / 4} \Delta\right)^{2} \operatorname{mes}\left(S_{h} \backslash R_{h}\right) \ll \frac{h T^{1 / 2}}{\log T} .
$$

Thus

On choosing

$$
\operatorname{mes}\left(S_{h} \backslash R_{h}\right) \ll \frac{h T}{\Delta^{2} \log T} .
$$

$$
\Delta=h(h \log T)^{-1 /(2 K+1)}
$$

we therefore deduce from (10) that

$$
\operatorname{mes}\left(S_{h}\right) \ll T(h \log T)^{-1+2 /(2 K+1)} .
$$

Our choice of $\Delta$ will satisfy the conditions (6) and (7) if $h=\left(a^{\prime} \log T\right)^{-1}$ with $h \leq T^{3 / 4}$ and $0<a^{\prime} \leq a^{\prime}(K)$, say.

We are now ready to estimate

$$
\sum_{\hat{\gamma}_{i}}\left(\hat{\gamma}_{i+1}-\hat{\gamma}_{i}\right)^{\mu}
$$

for $T \leq \hat{\gamma}_{i} \leq 2 T$. We shall choose $K$ to be a fixed integer such that

$$
\frac{2}{2 K+1}<2-\mu \text {. }
$$

According to a result of Hardy and Littlewood [2] we have $\hat{\gamma}_{i+1}-\hat{\gamma}_{i} \ll T^{\theta}$ for any $\theta>\frac{1}{4}$, so that it suffices to prove that

$$
\sum_{\hat{\gamma}_{i}}\left(\hat{\gamma}_{i+1}-\hat{\gamma}_{i}\right)^{\mu} \ll T(\log T)^{1-\mu}
$$

for $T \leq \hat{\gamma}_{i}<\hat{\gamma}_{i+1} \leq 2 T$. Moreover, summands for which

$$
\hat{\gamma}_{i+1}-\hat{\gamma}_{i} \leq \frac{8}{a^{\prime}(K) \log T}
$$

clearly make a satisfactory contribution. We shall classify the remaining terms according to the value of $h=2^{H}, H=1,2, \ldots$, for which

$$
4 h<\hat{\gamma}_{i+1}-\hat{\gamma}_{i} \leq 8 h .
$$


Thus we may assume that

$$
\frac{1}{a^{\prime}(K) \log T} \leq h \leq T^{3 / 4}
$$

Since $t \in S_{h}$ for $\hat{\gamma}_{i}+h \leq t \leq \hat{\gamma}_{i}+2 h$, we see that the number of points $\hat{\gamma}_{i}$ corresponding to any such $h$ is at most

$$
h^{-1} \operatorname{mes}\left(S_{h}\right) \ll h^{-2+2 /(2 K+1)} T(\log T)^{-1+2 /(2 K+1)} .
$$

The corresponding contribution to (11) is therefore

$$
\ll h^{\mu-2+2 /(2 K+1)} T(\log T)^{-1+2 /(2 K+1)},
$$

and summing over $h=2^{H} \gg(\log T)^{-1}$ yields the required result.

\section{References}

[1] Fujii, A.: On the distribution of the zeros of the Riemann Zeta function in short intervals. Bull. Amer. Math. Soc. 81, 139-142 (1975)

[2] Hardy, G.H., Littlewood, J.E.: Contributions to the theory of the Rieman zeta-function and the theory of the distribution of primes. Acta Math. 41, 119-196 (1918)

[3] Selberg, A.: On the zeros of Riemann's zeta-function. Skr. Norske Vid. Akad. Oslo no. 10, (1942)

[4] Titchmarsh, E.C.: The theory of the Riemann Zeta-function, 2nd Edition, revised by D.R. Heath-Brown. Oxford: Clarendon Press 1986 